\title{
Cystic Echinococcosis of Liver and Spleen Communicating to the Lung: A Rare Case
}

\author{
Jitu Mani Kalita ${ }^{1}$ Palanisamy Naveenraj ${ }^{2}$ Vidhi Jain ${ }^{1}$ Deepak Kumar ${ }^{2}$ Vibhor Tak ${ }^{1}$ \\ Pawan Kumar Garg ${ }^{3}$ Vijaya Lakshmi Nag ${ }^{1}$
}

${ }^{1}$ Department of Microbiology, All India Institute of Medical Sciences, Jodhpur, Rajasthan, India

2 Department of General Medicine, All India Institute of Medical

Sciences, Jodhpur, Rajasthan, India

${ }^{3}$ Department of Diagnostic and Interventional Radiology, All India

Institute of Medical Sciences, Jodhpur, Rajasthan, India
Address for correspondence Vibhor Tak, MD, Department of Microbiology, All India Institute of Medical Sciences, Jodhpur, Rajasthan, 342005, India (e-mail: vibhor_tak@yahoo.com).

J Lab Physicians 2022;14:351-354.

\begin{abstract}
Keywords

- cystic echinococcosis

- Echinococcus granulosus

- albendazole

- parasitic zoonosis

The annual cystic echinococcosis (CE) incidence in endemic countries is between 1 and 200 per 100,000 population. The clinical manifestations of CE are associated with damage or dysfunction of target organs and affect primarily the liver in around $70 \%$ of cases and lungs in around $20 \%$ of cases. The involvement of the spleen is rarely seen, representing 2 to $6 \%$ of all CE cases. We describe a 60 -year-old female patient who presented with 15 days' history of intermittent fever and abdominal distension, having significant hepatosplenomegaly. CE was diagnosed involving the liver, spleen, and lungs and was confirmed radiologically and microbiologically. CE is caused by the larval or cystic stage of the zoonotic dog-tapeworm. Humans can be infected by ingestion of contaminated food or water. At present, there are nine species under the genus Echinococcus with $E$. granulosus sensu stricto having worldwide distribution, while the rest are focally distributed. CE involving multiple organs is rare. CE should be taken into consideration in patients with these symptoms in endemic areas.
\end{abstract}

\section{Introduction}

Echinococcosis is a parasitic infectious disease and can present in two forms: cystic echinococcosis (CE) caused by Echinococcus granulosus sensu lato and alveolar echinococcosis caused by Echinococcus multilocularis. ${ }^{1}$ CE is more prevalent worldwide except for a few island countries that have eliminated it. ${ }^{2,3}$ The annual CE incidence in endemic countries is between 1 and 200 per 100,000 population. ${ }^{4}$ The mortality rate due to $C E$ ranges from 2 to $4 \%$ but may increase if adequate treatment is not provided. The clinical manifestations of CE are associated with damage or dysfunction of target organs and affect primarily the liver in around $70 \%$ of cases and lungs in around $20 \%$ of cases. Other organs that can be involved are the brain, spleen, kidneys, heart, and muscles. Generally, the disease progresses very slowly and the patient may remain asymptomatic for 10 to 15 years. Symptoms usually appear when the cyst becomes large in size ( $>10 \mathrm{~cm}$ in diameter) or when more than $70 \%$ of the organ volume is occupied by the cyst or cysts. ${ }^{5}$ Simultaneous involvement of liver and lung has been rarely reported in international literature and may constitute around 5 to $13 \%$ of all cases. ${ }^{6}$ The involvement of the spleen is also rare, published online February 15, 2022
DOI https://doi.org/

$10.1055 / \mathrm{s}-0042-1742416$ ISSN 0974-2727. (c) 2022. The Indian Association of Laboratory Physicians. All rights reserved.

This is an open access article published by Thieme under the terms of the Creative Commons Attribution-NonDerivative-NonCommercial-License, permitting copying and reproduction so long as the original work is given appropriate credit. Contents may not be used for commercial purposes, or adapted, remixed, transformed or built upon. (https://creativecommons.org/ licenses/by-nc-nd/4.0/)

Thieme Medical and Scientific Publishers Pvt. Ltd., A-12, 2nd Floor, Sector 2, Noida-201301 UP, India 
representing 2 to $6 \%$ of all CE cases. ${ }^{7}$ Here, we are reporting a case of CE involving the liver and spleen and communicating to the lungs.

\section{Case Report}

A 60-year-old female patient came to the emergency with 15 days history of intermittent fever and abdominal distension. On examination, significant hepatosplenomegaly was present. Based on clinical history, physical examination, and endemicity, a provisional diagnosis of enteric fever was made and injection ceftriaxone (1 gm i.v. BD) was started as an empirical therapy after sending appropriate blood cultures. RT-PCR test was negative for SARS-CoV-2 and it was performed as per the mandatory inpatient screening policy of our institute during the COVID-19 pandemic. Routine workup for acute febrile illnesses was done and her serum was found to be reactive for Salmonella typhi IgM antibody by Typhidot IgM/IgG rapid antibody detection test. She was tested negative for malaria and dengue. Chest X-ray showed left lower zone opacity suggestive of pleural effusion. Ultrasonography (USG) abdomen was suggestive of hydatid cyst with left-sided moderate pleural effusion (empyema) with underlying collapse consolidation having the possibility of infection or continuity of the splenic lesion. The description of the mass in USG was well defined, heteroechoic mass $(9.9 \times 7.9 \times 10.3 \mathrm{~cm})$ in the retroperitoneum. Tablet albendazole ( $400 \mathrm{mg} \mathrm{BD}$ ) was added to the therapy after the USG report. Due to inconclusive USG report and to rule out tuberculosis and other bacterial infections, USG-guided as-

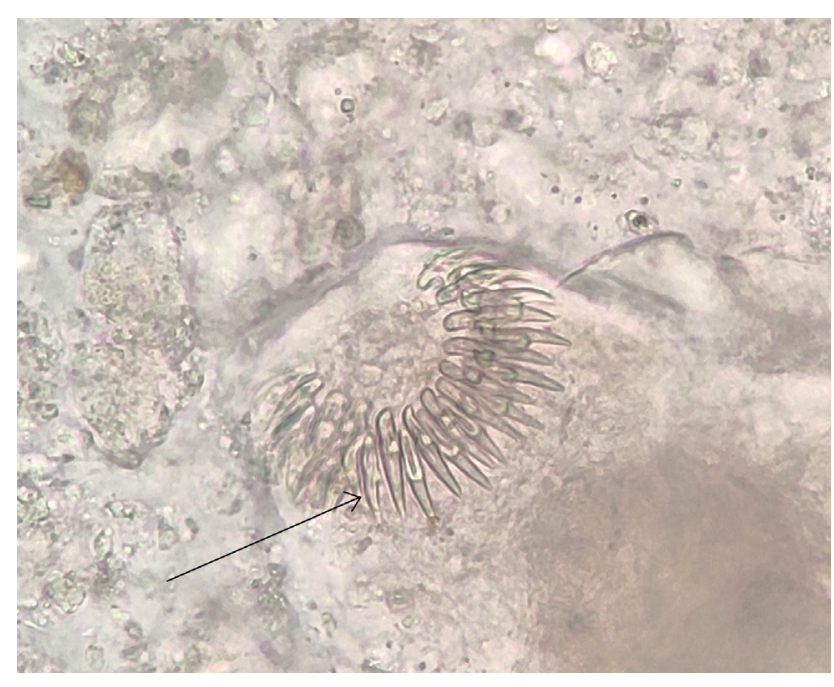

Fig. 1 Unstained wet mount preparation of pleural fluid showing hooklets of Echinococcus granulosus sansu lato (black arrow).

piration of pleural fluid was done and it was negative for microorganisms on Gram staining, aerobic culture, ZiehlNeelsen staining, and mycobacterial culture. It was further subjected to wet mount examination. An unstained preparation showed hooklets of Echinococcus granulosus sansu lato (-Fig. 1). CT scan of abdomen and thorax revealed multiple cystic lesions in the liver and spleen with dense peripheral calcifications, suggestive of hydatid disease ( - Fig. 2A and B) (WHO stage CE5) along with loculated collection with peripheral enhancement at the peripheries of the left lower

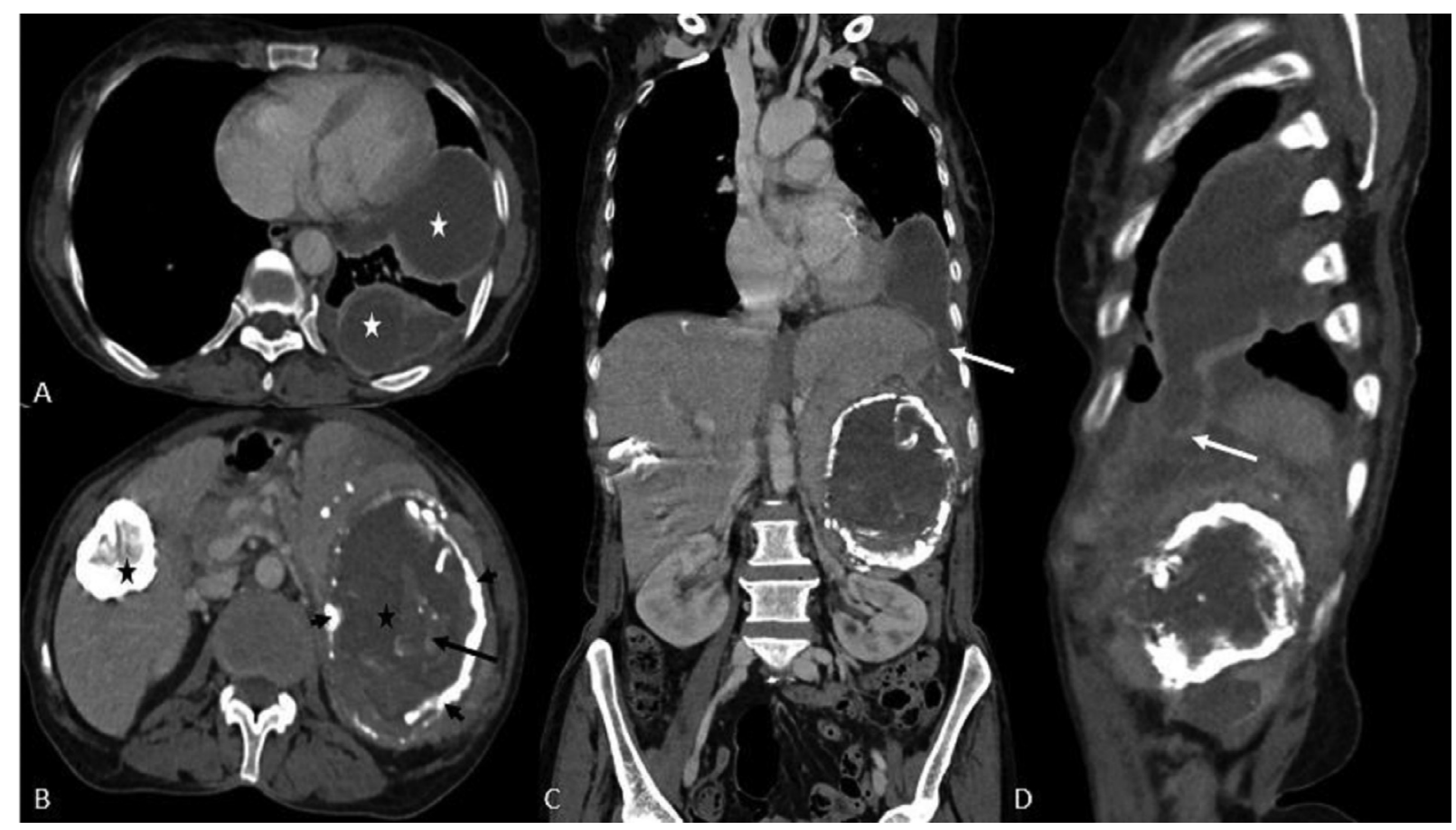

Fig. 2 Contrast-enhanced computed tomography (CT) axial (A, B), coronal (C), and sagittal (D) images showing multiple hydatid cysts. (Lesions are in the left lower part of the pleural space [white asterisk], liver, and the spleen [black asterisk]). The lesion in the liver is completely hyperdense, suggestive of a calcified cyst. The splenic lesion is showing peripheral discontinuous calcification (short black arrow) in the cyst wall with the presence of an internal membrane (long black arrow) and dependent debris. The lesion in the spleen is ruptured and reached the left side of the pleural space through the diaphragm (white arrow). 
lobe of lungs abutting the mediastinal as well as coastal pleura suggestive of infected collection, with possible continuity with the splenic parenchymal lesions ( - Fig. 2 C and D). As the pleural tap was purulent, intercostal drainage tube insertion was planned initially; however, it was stopped because of the presence of parasite structure in the pleural fluid. IgG antibody against Echinococcus species was also positive. The patient reported a history of close association (rearing, milking, feeding) with cattle. However, there was no history regarding close association with dogs. Complete blood count and liver function test revealed no abnormalities except for low hemoglobin level $(4.9 \mathrm{~g} / \mathrm{dL})$. Further investigations were suggestive of nutritional anemia. ESR was $12 \mathrm{~mm}$ /hour (Westergren). HBsAg, anti-HCV, and HIV 1 and 2 antibody tests were negative. Her blood culture was also sterile. After radiological and microbiological confirmation of the diagnosis, gastrosurgery consultation was taken and planned for surgery for the pleural collection but the patient did not give consent for surgery and left the hospital against medical advice. At the time of discharge, tablet albendazole (400 mg BD) was prescribed for 1 month and advised for follow-up in medicine OPD.

\section{Discussion}

CE is caused by the larval stage of the zoonotic dog-tapeworm. ${ }^{8}$ Humans can be infected by ingestion of contaminated food or water. Echinococcus granulosus was considered a single species but it is now recognized as having distinct strains or genotypes having differences in disease pathology as well as differences in response to therapy. ${ }^{5}$ Ten genotypes (G1-G10) have been recognized based on the mitochondrial DNA sequencing of the parasite. ${ }^{9}$ E. granulosus sensu lato have been further divided into five species: E. granulosus sensu stricto (the former sheep strain), E. ortleppi (cattle strain), E. equinus (horse strain), E. canadensis (camel strain/pig strain/cervid strains), and E. felidis (lion strain). ${ }^{10,11}$ At present, there are nine species under the genus Echinococcus with E. granulosus sensu stricto having worldwide distribution, and the rest are focally distributed. ${ }^{5}$ After infection, the involvement of the liver is through the portal venous system or lymphatic system, and without treatment, dissemination can occur to other organs such as lungs, kidneys, bones, and brain. ${ }^{12}$ Rarely affected organs by the parasites are the heart, spleen, pancreas, and muscles. An increase in the size of the hydatid cyst generally results in physical compression or damage to the adjacent structures. CE involving liver or spleen can present with upper abdominal discomfort and in case of a large liver cyst, clinical icterus may result due to compression of the bile duct. On examination, a tumor-like mass in the abdomen, hepatomegaly, splenomegaly, or abdominal distension may be found as in the present case. If the lungs are involved, the patient may present with chest pain, cough, or hemoptysis. However, in the present case, the patient had only a mild cough. In any organ, if a rupture of cyst occurs, the patient may present with sudden onset fever, urticaria, eosinophilia, or can cause anaphylactic shock. Because of the potential risk for life- threatening allergic reactions, any diagnostic or therapeutic tapping of a hydatid cyst is contraindicated. In the present case, cystic lesions were absent in the lungs; however, a collection was present in the left lower lobe. It was aspirated under USG guidance to rule out a bacterial infection in which the parasite structure was seen. This patient did not develop any allergic reactions. Splenic echinococcosis has been reported rarely and it can occur in patients with portal hypertension or rupture of the hepatic cyst through retrograde spread from the liver via the hepatic, portal, and splenic veins. ${ }^{13}$ Approximately $30 \%$ of splenic hydatid cysts are asymptomatic. They usually grow at a very slow rate (2$3 \mathrm{~cm} /$ year) and the patient may remain asymptomatic for years before diagnosis; so, diagnosis may be incidental. ${ }^{14}$ Without treatment, the patient can present with various complications such as rupture of the cyst, secondary infection, fistula formation into adjacent organs, pleural effusion, empyema, hypersplenism, and severe urticaria. In the present case, the patient had pleural effusion that radiologically (on CT scan) appeared to be a possible continuation of the splenic lesion. Radiological investigations such as USG and CT scans of the abdomen are sensitive tools for the diagnosis of $\mathrm{CE}$ and appearances of the cysts in imaging, depending on the stage of cyst development. ${ }^{8}$ Some imaging characteristics that can be seen mostly are calcification of the cyst wall, presence of daughter cysts, and membrane detachment. ${ }^{15} \mathrm{CT}$ scan can provide additional structural details than USG with precise location and depth of the cyst within the organ involved. The sensitivity and specificity of serological methods are reported to be 60 to $90 \%$ in confirmed CE cases by surgical resection. Currently, serology is used only for confirmation of imaging results. The WHO-informal working group on echinococcosis (IWGE)-Expert consensus has included serology results in the definition of possible and probable echinococcosis cases. ${ }^{5}$ Curative treatment of $\mathrm{CE}$ is achieved by the complete removal of the cyst irrespective of its location. In subtotal cystectomy, all types of partial cystectomy, and in percutaneous "PAIR" (puncture, aspiration, injection, and respiration) technique, protoscolicidal agents should be used following the therapeutic procedure. The only systemic anti-infective treatments that have proven efficacy are two benzimidazole carbamates, albendazole, and mebendazole. Albendazole is preferred as an anti-infective treatment of choice due to its increased bioavailability and easier administration to patients, at an average dosage of $15 \mathrm{mg} / \mathrm{kg} /$ day. ${ }^{5}$ In the present case, the patient did not give consent for surgery and tablet albendazole was advised.

Cystic echinococcosis with multiple organ involvement is rare. The present case, who presented with fever and hepatosplenomegaly found to have CE involving the liver, spleen, and lungs. CE should be taken into consideration in patients with these symptoms in endemic areas.

\section{Funding \\ None.}

Conflict of Interest

None declared. 
354 Cystic Echinococcosis of Liver and Spleen Communicating to the Lung Kalita et al.

\section{References}

1 McManus DP, Gray DJ, Zhang W, Yang Y. Diagnosis, treatment, and management of echinococcosis. BMJ 2012;344:e3866

2 Craig PS, Larrieu E. Control of cystic echinococcosis/hydatidosis: 1863-2002. Adv Parasitol 2006;61:443-508

3 Craig PS, McManus DP, Lightowlers MW, et al. Prevention and control of cystic echinococcosis. Lancet Infect Dis 2007;7(06):385-394

4 Schweiger A, Ammann RW, Candinas D, et al. Human alveolar echinococcosis after fox population increase, Switzerland. Emerg Infect Dis 2007;13(06):878-882

5 Wen H, Vuitton L, Tuxun T, et al. Echinococcosis: advances in the 21st century. Clin Microbiol Rev 2019;32(02):e00075-e18

6 Shah NJ, Vithalani NK, Chaudhary RK, Mohite PN. Disseminated peritoneal hydatidosis following blunt abdominal trauma: a case report. Cases Journal 2008;1:118. Doi: 10.1186/1757-1626-1-118

7 Mamishi S, Sagheb S, Pourakbari B. Hydatid disease in Iranian children. J Microbiol Immunol Infect 2007;40(05):428-431

8 Geller DA, Goss JA, Tsung ALiver. In: Brunicardi FC, Andersen DK, Billiar TRet al, eds. Schwartz's Principles of Surgery. 9th ed. New York: McGraw-Hill; 2010:1116
9 McManus DP. Molecular discrimination of taeniid cestodes. Parasitol Int 2006;55(Suppl):S31-S37

10 Alvarez Rojas CA, Romig T, Lightowlers MW. Echinococcus granulosus sensu lato genotypes infecting humans-review of current knowledge. Int J Parasitol 2014;44(01):9-18

11 Nakao M, Lavikainen A, Yanagida T, Ito A. Phylogenetic systematics of the genus Echinococcus (Cestoda: Taeniidae). Int J Parasitol 2013;43(12-13):1017-1029

12 Ionescu A, Jakab A, Jutis T, Forai F, Ota A. [Splenic hydatid cyst]. Rev Med Chir Soc Med Nat Iasi 1990;94(3-4):525-528

13 Motie MR, Rezapanah A, Pezeshki Rad M, Razavian H, Azadmand A, Khajeh M. Primary localization of a hydatid cyst in the latissimus dorsi muscle: an unusual location. Surg Infect (Larchmt) 2011;12(05):401-403

14 Malik AA, ul Bari S, Younis M, Wani KA, Rather AA. Primary splenic hydatidosis. Indian J Gastroenterol 2011;30(04): 175-177

15 Karabicak I, Yurtseven I, Yuruker SS, Ozen N, Kesim M. Splenic hydatid cyst. Can J Surg 2009;52(05):E209-E210 